\title{
AC 2007-414: FINITE ELEMENT MODULES FOR ENHANCING UNDERGRADUATE TRANSPORT COURSES: APPLICATIONS TO FUEL CELL FUNDAMENTALS
}

Jason Keith, Michigan Technological University Jason Keith is an Associate Professor of Chemical Engineering at Michigan Technological University. He recieved his PhD from the University of Notre Dame in 2000. Jason teaches the required Transport / Unit Operations 2 course and an elective in fuel cell fundamentals.

Faith Morrison, Michigan Technological University

Faith Morrison is an Associate Professor of Chemical Engineering at Michigan Technological University. She recieved her PhD from the University of Massachusetts in 1988. Faith teaches the required Transport / Unit Operations 1 course and electives in polymer rheology. She is author of the textbook Understanding Rheology.

\section{Julia King, Michigan Technological University}

Julia King is an Associate Professor of Chemical Engineering at Michigan Technological University. She recieved her PhD from the University of Wyoming in 1989. Julie teaches the required Transport / Unit Operations 1 and Fundamentals of Chemical Engineering 1 courses. 


\title{
Finite Element Modules for Enhancing Undergraduate Transport Courses: Application to Fuel Cell Fundamentals
}

\begin{abstract}
The transport phenomena courses (momentum, heat, and mass transfer) in chemical engineering typically contain many mathematical derivations and may often lack practical applications. The use of finite element software can help students visualize solutions and see how parameter changes affect velocity, temperature, and concentration profiles and their corresponding fluxes for design of practical systems.
\end{abstract}

Alternative energy is a rapidly growing research area yet is lacking in available course content for chemical engineering transport phenomena. In this paper we illustrate the use of the finite element method using Comsol Multiphysics ${ }^{1}$ (formerly known as FEMLAB) for problems related to the design of fuel cells and their components. As such, we present ready-made tutorials for use in undergraduate transport courses.

Introduction and Motivation: The Typical Transport Course

As is the case with many core courses in the undergraduate curriculum, courses in fluid mechanics, heat transfer, and mass transfer can be categorized into three generic classifications:

1. Transport phenomena approach - a highly theoretical approach focusing on the derivation of microscopic conservation equations and their solutions, such as that contained in the text of Bird, Stewart, and Lightfoot ${ }^{2}$.

2. Unit operations approach - a highly practical approach focusing on macroscopic balance equations and using them for the design of pumps, heat exchangers, and membranes, such as that contained in the text of McCabe, Smith, and Harriott ${ }^{3}$.

3. A balance between the transport phenomena and unit operations, such as that contained in the text of Geankoplis ${ }^{4}$.

At Michigan Technological University, students must complete a two-semester sequence of lecture courses (CM 3110 Transport / Unit Operations 1; CM 3120 Transport / Unit Operations 2). Based upon the title of the course we typically follow the third classification; however, content can vary depending on the instructor.

In a recent ASEE paper, Krantz discussed that the above textbooks often focus on simple problems with analytical or numerical solutions, but the development of software for performing computational analysis has allowed instructors of transport phenomena to focus on model development by introducing more complex problems ${ }^{5}$. An additional advantage of the software is that it allows the students to visualize the transport processes taking place.

Other studies have also used computers to help students learn concepts in chemical engineering education. This includes that of Thompson ${ }^{6}$, who has used the partial 
differential equation (PDE) toolbox within MATLAB to visualize steady laminar flow in a finned heat exchanger, transient and steady heat transfer in a finned heat exchanger, and wave propagation in a heterogeneous material. Sinclair ${ }^{7}$ has used FLUENT computational fluid dynamics software within the undergraduate curriculum. Besser ${ }^{8}$ has used EXCEL spreadsheets to study two-dimensional heat conduction in solid materials. Zheng and Keith ${ }^{9-10}$ have developed JAVA applets for unsteady and steady state transport problems.

As such, we present a handful of problems developed with the Comsol Multiphysics (formerly known as FEMLAB) finite element method modeling software ${ }^{1}$. We also use the "Chemical Engineering Module" which allows for quick access to the typical governing equations of momentum, heat, and mass transport. Additional modules are also available.

As the authors are working on a National Science Foundation project to develop new materials for fuel cell bipolar plates, many of the modules developed here focus solving a variety of fluid mechanics, heat transfer, and mass transfer problems applied to the relatively modern field of fuel cells.

After a very brief overview of fuel cells, six modules are presented that may be of use to instructors of transport phenomena courses.

\section{Fuel Cell Overview}

A fuel cell is device that converts a fuel into electricity with heat as a byproduct. There are several types of fuel cells, with the most likely fuel cell to be used for transportation applications being the proton exchange membrane fuel cell. In this device, the hydrogen fuel reacts with oxygen from the air and produces water. A single cell of a fuel cell produces about $0.7 \mathrm{~V}$ of potential; for many applications the cells are "stacked" together to give a higher voltage to power an electric motor. As such, the majority of design and analysis of fuel cell systems focuses on a single cell. A cartoon is shown in figure 1 below. 


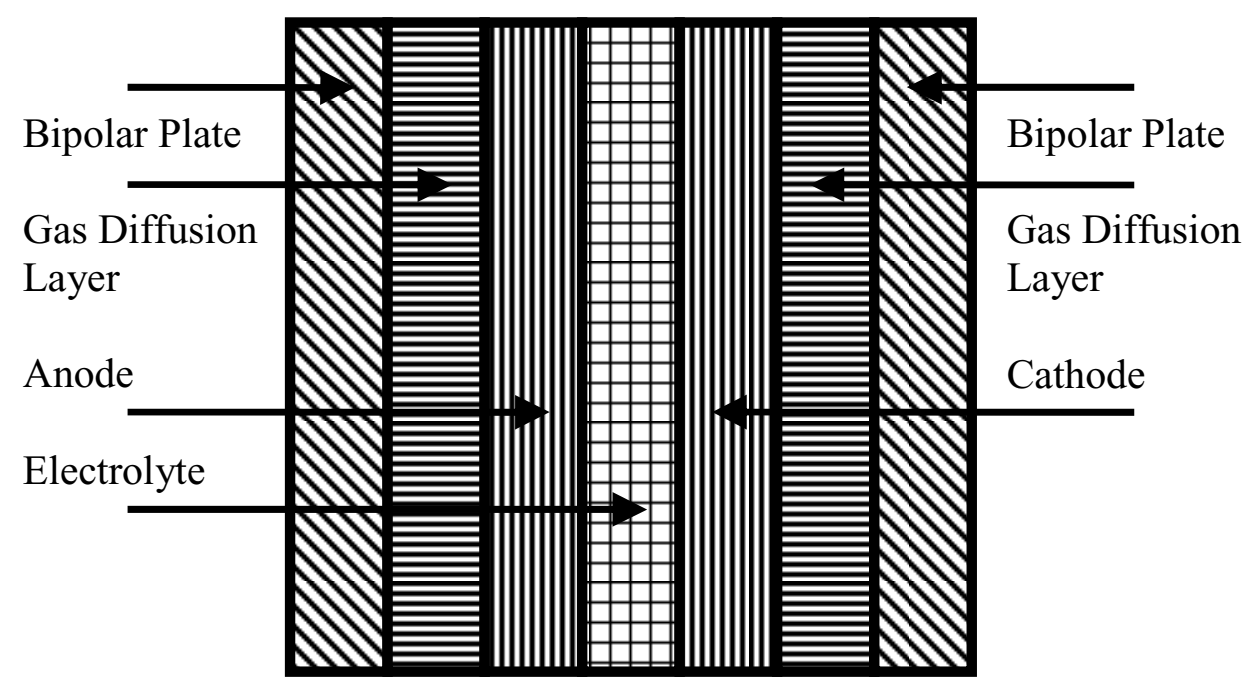

Figure 1. Schematic of one cell of a proton exchange membrane fuel cell. The slanted lines are the bipolar plates, the horizontal lines are the gas diffusion layer, the vertical lines are the electrodes (left block is the anode; right block is the cathode), and the grid represents the electrolyte.

Within a single cell of a fuel cell are bipolar plates which function to separate one cell from the other. The bipolar plates have channels etched on either side to allow for reactant and product gases to flow. The plates also need to have low hydrogen permeation, high thermal conductivity, and high electrical conductivity. Within the channels the chemicals reach a gas diffusion layer, and are transported through this layer, after which where they encounter the electrodes. The electrodes contain a platinum catalyst which facilitates the conversion of the fuel into protons and electrons. The protons pass through a sulfonated polymer electrolyte membrane. Meanwhile, the electrons are conducted back through the gas diffusion layer, bipolar plate, and electric load where they react with the protons and oxygen to form water. For more information regarding fuel cell construction, the reader is referred to the text of Larminie and Dicks ${ }^{11}$ or the Los Alamos National Laboratory fuel cell website ${ }^{12}$.

Finite Element Problems

In this paper we develop five modules in the following areas:

- Fluid Flow

O The first module concerns the flow of polymer melts in a capillary rheometer. We note that rheology needs to be understood for compression molding analysis of complex bipolar plate designs. The objective of the module is to determine the velocity profile for laminar and power-law fluids, measure the pressure drop, and compare with published correlations. The users can also calculate Reynolds numbers and entrance lengths for the velocity profile to develop. 
o The second module concerns the flow of gases in bipolar plate channels. We note that pressure drop and reactant uniformity is important in fuel cell systems to improve overall fuel conversion efficiency. The objective of the module is to create a model of a channel with complicated flow geometry (U-shaped section) and determine the pressure drop over this channel. The users are then asked to consider multiple sections and the design of plates with multiple flow channels.

- Heat Transfer

- The third module concerns steady-state one-dimensional heat conduction in a composite slab with reaction. We note that thermal analysis can allow for design of low or high temperature fuel cells. The objective of the module is to model multi-phase heat transfer and compare simulation results with the analytical solution.

- Mass Transfer

o The fourth module concerns one- and two-dimensional unsteady state diffusion in a slab. We note that bipolar plates have low hydrogen permeation, but a complete analysis allows for estimates of fuel cell overall efficiency. The objective of the module is to model mass transfer and compare simulation results with a cumbersome analytical Fourier series solution. The users are then asked to solve the unsteady-state diffusion equation in a complex geometry that applies to a real fuel cell bipolar plate.

- Transport Effects on Kinetics

o The fifth and final module concerns diffusion and reaction in a porous solid. We note that transport effects can have a large impact on fuel cell performance and catalyst design. This is the classical Thiele modulus problem where the user calculates concentration profiles and catalyst effectiveness factors for a cube-shaped and for a cone-shaped catalyst pellet.

Typical problems, shown here as separate appendices, walk the user through an example so they can become acquainted with how the software works. Many of these examples have analytical solutions so one can determine accuracy of the numerical model. This allows for discussions on computational methods and convergence.

At the conclusion of the modules there is often a question or portion where the users apply their knowledge to a more complex problem that usually cannot be solved analytically. It is noted that the first module includes several figures to illustrate the set up of the model geometry. All modules include figures which illustrate the results.

\section{Conclusions}

We present several ready-to-use modules using Comsol Multiphysics finite element software for the undergraduate transport courses. The topics can easily be integrated into a momentum, heat, or mass transfer course. Students using the finite element method benefit from enhanced visualization of the physical processes occurring and also benefit 
from seeing practical applications of the complex partial differential equations that are typically derived in these courses.

To date these modules have only been developed and some are currently being used in our courses. In a future paper we will assess student learning skills with these tools.

Acknowledgments

The authors would like to acknowledge the Department of Energy (Award Number DEFG02-04ER63821), the National Science Foundation (DMI-0456537), and the Michigan Space Grant Consortium for partially funding this project. The authors also thank the following undergraduate students for their assistance on this project: Emily Kunen, Peter Grant, and Joan Wierzba.

References

1. Comsol Multiphysics Modeling Website, http://www.comsol/com, accessed January 2007.

2. Bird, R. B.; Stewart, W. E.; Lightfoot, E. N. Transport Phenomena, $2^{\text {nd }}$ Edition, Wiley, New York, NY, 2002.

3. McCabe, W. L.; Smith, J. C.; Harriott, P. Unit Operations of Chemical Engineering, $6^{\text {th }}$ Edition, McGraw-Hill, New York, NY, 2001.

4. Geankoplis, C. J.; Transport Processes and Separation Process Principles, $4^{\text {th }}$ Edition, Prentice Hall, Upper Saddle River, NJ, 2003.

5. Kranz, W. B., "Pediment Graduate Course in Transport Phenomena," Proceedings of the 2003 American Society for Engineering Education Annual Conference \& Exhibition.

6. Thompson, K. E., "Teaching PDE-Based Modeling to ChE Undergraduates," Chemical Engineering Education, 34, 146 (2000).

7. Sinclair, J. L., "CFD Case Studies in Fluid-Particle Flow," Chemical Engineering Education, 32, 108 (1998).

8. Besser, R. S., "Spreadsheet Solutions to Two-Dimensional Heat Transfer Problems," Chemical Engineering Education, 34, 160 (2002).

9. Zheng, H.; Keith, J. M. "JAVA-Based Heat Transfer Visualization Tools," Chemical Engineering Education, 38, 282 (2004).

10. Zheng, H.; Keith, J. M. "Web-Based Instructional Tools for Heat and Mass Transfer," Proceedings of the 2003 American Society for Engineering Education Annual Conference \& Exhibition.

11. Larminie, J.; Dicks, A. Fuel Cell Systems Explained, $2^{\text {nd }}$ Edition, Wiley, West Sussex, England, 2003.

12. Los Alamos National Laboratory fuel cell website, http://www.lanl.gov/orgs/ee/fuelcells/index.shtml, accessed January 2007. 


\section{Example 1: Newtonian and Non-Newtonian Fluid Flow in a Capillary Rheometer}

Note: This example problem will be used in the required course "Transport / Unit Operations 1" which is taken by junior-level chemical engineering students.

Problem Statement: A capillary viscometer consists of a very small diameter, cylindrical capillary tube. A liquid is forced through the capillary by imposing a pressure drop. The very small diameter of the tube and the very large length to diameter ratio minimizes entrance and exit effects and ensures a fully developed velocity profile.

For all fluids, the shear stress at the pipe wall $\left(\tau_{\mathrm{wall}}\right.$ in units of $\left.\mathrm{Pa}\right)$ is given as

$\tau_{\mathrm{wall}}=(\Delta P R) /(2 L)=(\Delta P D) /(4 L)$

Where: $\Delta P=$ pressure drop across the capillary tube, $\mathrm{Pa}$

$D=$ inside capillary tube diameter, $\mathrm{m}$

$R=$ inside capillary tube radius, $\mathrm{m}$

$L=$ capillary tube length, $\mathrm{m}$

For a laminar, incompressible, Newtonian fluid, the shear rate at the circular pipe wall, $\dot{\gamma}_{\text {wall }}$ can be calculated from the microscopic balances ${ }^{4}$. The result is shown below.

$\dot{\gamma}_{\text {wall }}=(4 Q) /\left(\pi R^{3}\right)=(32 Q) /\left(\pi D^{3}\right) 8 V / D=4 V / R$

Where: $\mathrm{Q}=$ volumetric flow rate, $\mathrm{m}^{3} / \mathrm{s}$

$\mathrm{V}=$ average fluid velocity, $\mathrm{m} / \mathrm{s}$

The Hagan-Poiseuille equation can also be calculated from the microscopic solution for this problem ${ }^{4}$, and may be used to calculate the viscosity of a laminar, Newtonian, incompressible fluid

$$
V=\frac{\Delta P R^{2}}{8 \mu L}
$$

The equations for Reynolds number and entrance length ${ }^{2}, L_{e}$, are shown below.

$$
\begin{aligned}
\mathrm{Re} & =\frac{D V \rho}{\mu} \\
L_{e} & =0.035 D \mathrm{Re}
\end{aligned}
$$

If you plot $\tau_{w}$ as a function of $\dot{\gamma}_{\text {wall }}$ for a Newtonian fluid, the slope is the viscosity of the fluid. 
$\tau_{\mathrm{wall}}=\mu \dot{\gamma}_{\text {wall }}$

Where: $\mu=$ viscosity of fluid (Pa-s)

For a power law fluid, you still use equation 1 to determine $\tau_{\text {wall. }}$ However, the following equation is used to relate wall shear stress and shear rate at the wall ${ }^{4}$ :

$\tau_{\text {wall }}=\mathrm{m}\left(\dot{\gamma}_{\text {wall }}\right)^{\mathrm{n}}$

Where: $\mathrm{m}=$ flow consistency index, $\mathrm{Pa}-\mathrm{s}^{\mathrm{n}}$, noting that $\mathrm{m}=\mu=$ viscosity of fluid if the fluid is a Newtonian fluid (The fluid is Newtonian when $\mathrm{n}=1$ )

$\mathrm{n}=$ flow behavior index, dimensionless

When $\mathrm{n}>1$ the fluid is shear-thickening (also called dilatant) meaning that as the shear rate is increased the fluid becomes more viscous, and when $n<1$ it is shear-thinning (also called pseudoplastic) meaning that as the shear rate is increased the fluid becomes less viscous.

In this module we are going to model the flow of water through a capillary rheometer with a radius of $0.5 \mathrm{~mm}$ and a length of $20 \mathrm{~mm}$. The fluid enters the tube at an average velocity of $0.0125 \mathrm{~m} / \mathrm{s}$ downward and exits at a pressure of $0 \mathrm{~Pa}$. From the module you will be able to determine the pressure drop over the length of the tube and you will view the velocity profile at the exit of the tube. You can then rerun the model for a nonnewtonian fluid: Vectra A950RX Liquid Crystal Polymer, which has potential use as a matrix material for recyclable fuel cell bipolar plates.

\section{Part 1: Start the program}

1.Start Comsol Multiphysics

2.Within the "New" tab of the "Model Navigator" window there should be a pull-down menu labeled "Space Dimension." Within this pull-down menu select "Axial Symmetry (2D)."

3. Expand the folder "Chemical Engineering Module" by clicking the "+" sign to the left of the folder followed by the same for Momentum Balance, Non-Newtonian Flow, and then select Steady-State Analysis. Click "OK." The program should then open to the main screen of the program with a red, vertical axis on it.

\section{Part 2: Create the domain}

In this module, a fluid will be modeled flowing through a capillary tube. The domain will be half of the vertical cross section of this tube, which when rotated completely around the z-axis creates the entire tube.

1.To draw a tube, select "Draw" from the tool bar and then "Specify Object" and select rectangle.

2. A box to specify the rectangle should appear on the screen as in the figure to the left below. For this problem we will enter length in meters, time in seconds, and mass in kilograms. Enter 5e-4 for "Width" (corresponding to the tube radius) and 2e-2 for 
"Height" (corresponding to the tube length) in the window that appears. Click "OK." The rectangle that appears represents a tube with a length-to-diameter of 20.
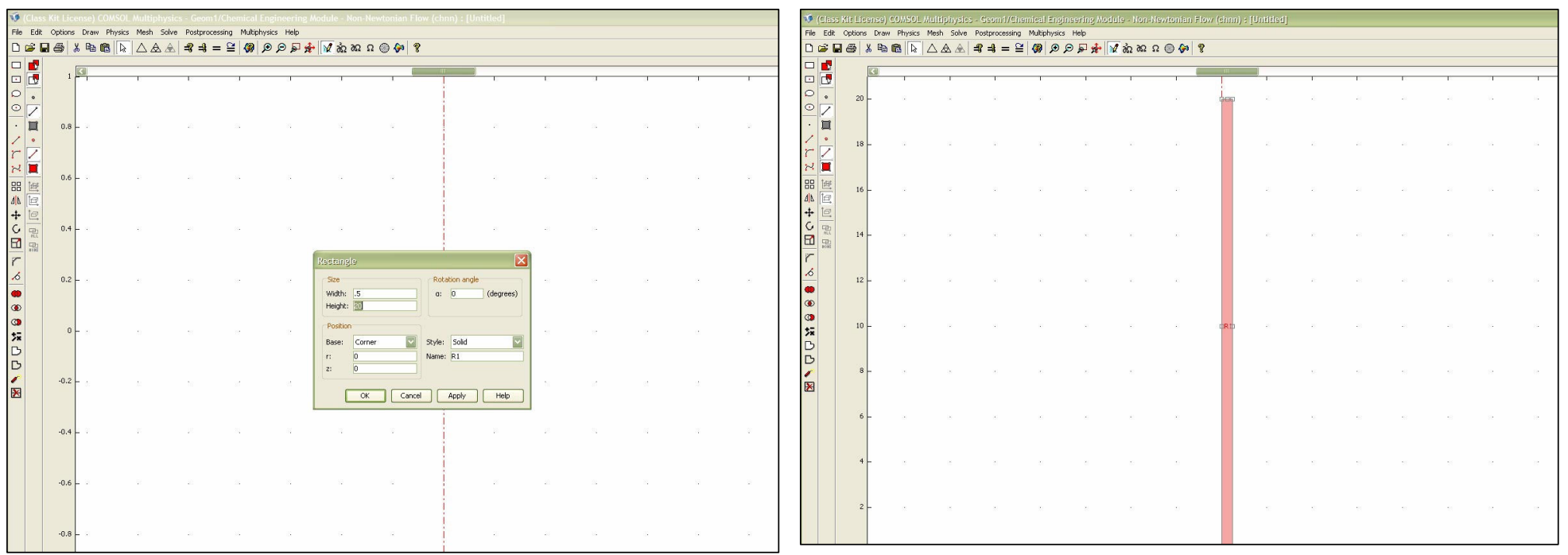

Figure 1.1. Setting up the model geometry.

3. Click the "Zoom Extents" button on the main toolbar. It looks like a red cross with a magnifying glass. This allows the user to view the entire geometry, as seen in the figure on the right above.

\section{Part 3: Apply physical properties}

1. From the top menu, select Physics, then Subdomain Settings

2. On the left hand side of the pop-up window, there will be a box labeled "Subdomain Selection." In this box select the subdomain (1).

3. On the right hand side of the pop-up window (under the "General" tab there is a box labeled "Fluid properties and sources/sinks." The third item from the top is Viscosity Model; make sure that the pull-down menu is set to "Power Law."

4. Switch from the "General" tab to the "Power Law" tab, then enter $\mathrm{m}=1 \mathrm{e}-3$ corresponding to the viscosity of water in $\mathrm{kg} / \mathrm{m}$-s and 1 as the $\mathrm{n}$ value to specify the Newtonian flow

5. Click "OK", or repeat steps if there are multiple subdomains.

\section{Part 4: Apply boundary conditions}

1. From the top menu, select Physics, then Boundary Settings. To specify boundaries highlight a number in the left portion of the box that appears (as shown below).

2. For the right edge of the geometry (edge that is not on the center line), select boundary condition "No Slip." 


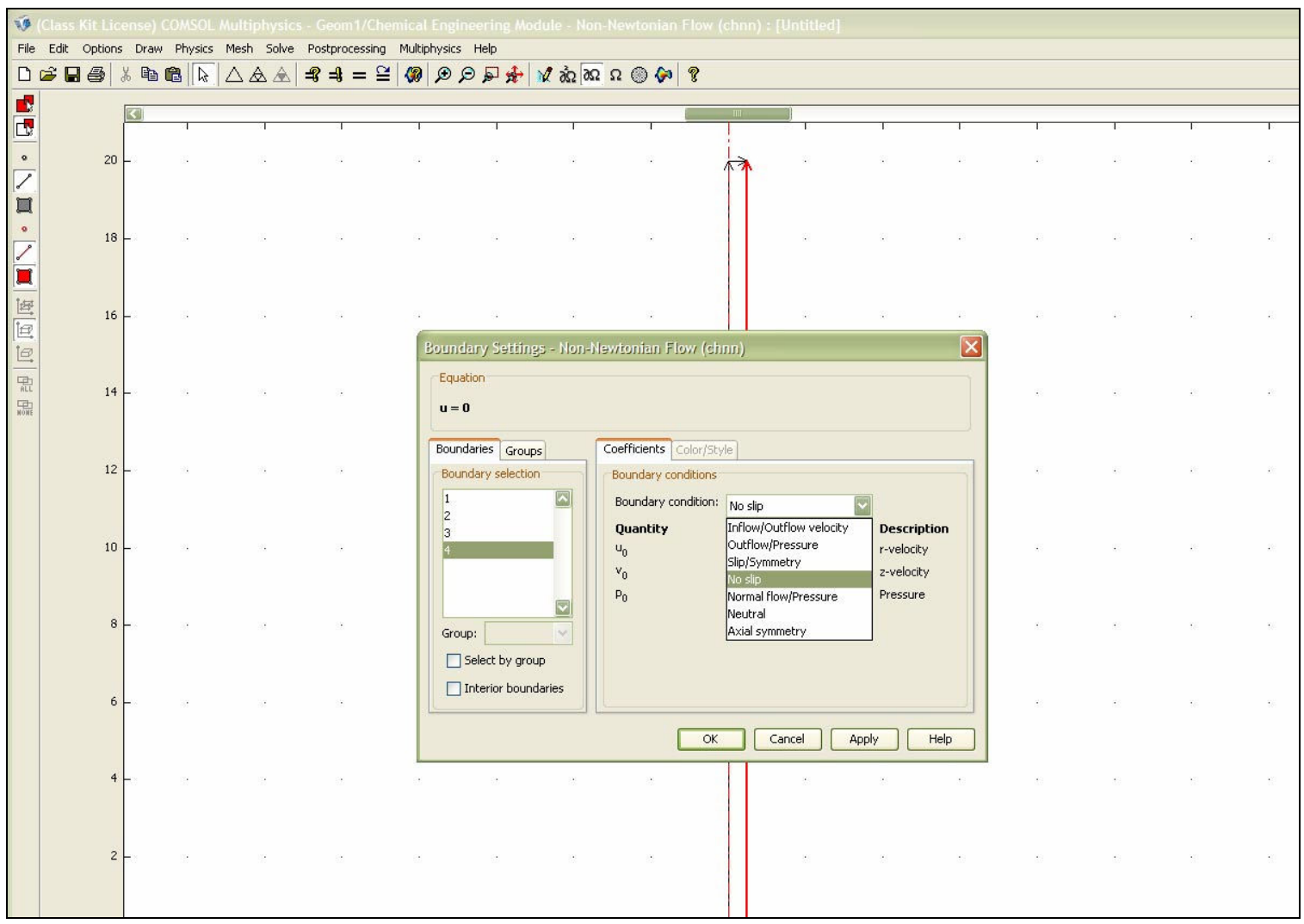

Figure 1.2. Setting boundary conditions.

3. For the axial side of the tube (edge that is on the center line), select the boundary condition "Axial Symmetry."

4. For the top boundary select "Inflow/Outflow Velocity" and enter $v_{0}=-0.0125$ (Description: average z-velocity in $\mathrm{m} / \mathrm{s}$ ) for a uniform downward flow at the inlet. This inflow velocity corresponds to a shear rate of $100 \mathrm{~s}^{-1}$.

5. For the bottom boundary (the outlet of the tube) select "Outflow/Pressure" and enter $p_{0}=0$ in $\mathrm{Pa}$. We are interested in the pressure drop over the length of the capillary tube, so 0 is used as a reference pressure, rather than the atmospheric pressure that would actually appear at the outlet of the tube. Click "OK."

\section{Part 5: Create the mesh}

1. From the top menu, click on the hollow triangle to initialize the mesh. In the bottom left of the screen it should say there are 120 elements in the mesh

2. The button next to "Initialize Mesh" is called "Refine Mesh" (which appears as a triangle within a triangle). This button takes the current mesh and simply makes it more detailed. Click the button once to make the mesh more refined. There should be 480 elements. Please note that problems may occur depending on graphics capabilities of your computer. If this occurs start over but do not refine the mesh.

\section{Part 6: Solve}

1.Click "Solve" from the pull-down menu and select "Solver Parameters" 
2. On the left hand side of the pop-up window there will be a box labeled "Solver:." Make sure that "Stationary nonlinear" is selected. Click "OK."

3 . From the top menu, click the "=" sign to solve. A window will appear showing how much progress has been made in solving the problem. After the problem is solved and the window disappears, you should see the geometry with a color spectrum indicating a velocity-concentration profile, as seen in the figure below. If you don't see anything, click on "Zoom Extents." This plot is called a Surface Plot. Notice the velocity is zero at the boundary and maximum at the center.

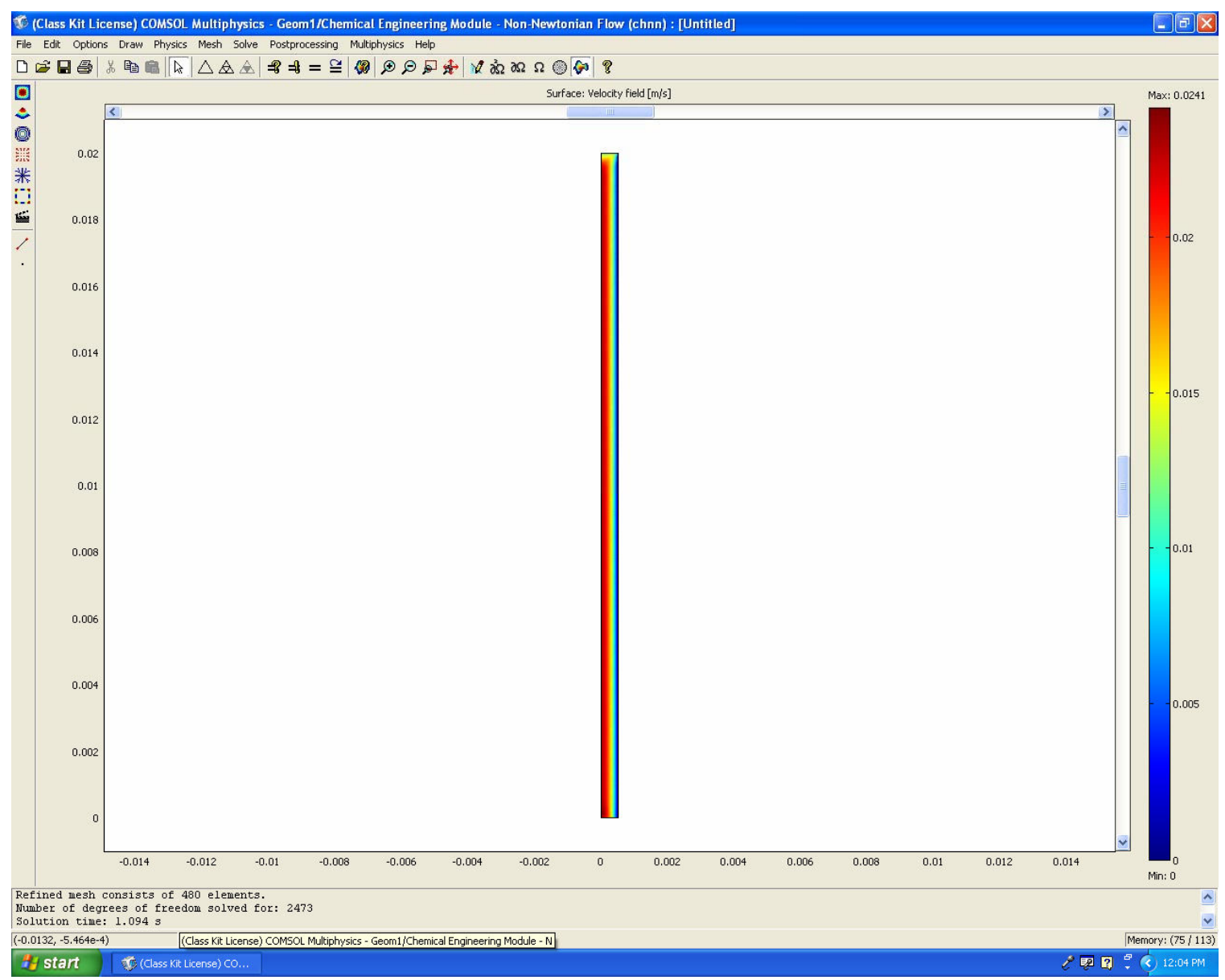

Figure 1.3 Velocity surface plot for water.

\section{Part 7: Post-Processing}

In this part you will produce a plot with the pressures at the inlet and outlet of the tube, and therefore be able to estimate the pressure drop across the length of the tube. You can compare this value to the change in pressure as calculated with the Hagen-Poiseuille law. You will also produce a plot of the velocity profile at the outlet of the tube.

1. From the top menu, select "Postprocessing" then "Cross-sectional plot parameters..." 2. Under the "Line/Extrusion" tab, in the "y-axis data" section, from the "Predefined Quantities" pull down menu, select Pressure as the desired field. 
3. In the "Cross-section Line Data section" select the coordinates between which you would like the measurements to be made. You want to do this once for the pressure at the top of the tube and once at the bottom, at a constant $\mathrm{z}$ value to see the profile across the tube radially - that is at a height of 0.0 and 0.02 . First, for the outlet, use $\mathrm{r} 0=0, \mathrm{r} 1=5 \mathrm{e}-4$, $z 0=1 e-6$, and $z 1=1 e-6$. These values are sufficiently close to zero, but still within the domain of interest. Under the "General" tab, select "keep current plot." Hit "OK" to complete a calculation of the pressure across the exit of the tube. Then, for the inlet repeat the calculation, starting again with selecting "Postprocessing" in step 1, using $z 0=0.019995$ and $z 1=0.0199995$. You should now have a plot of the pressures at the inlet and outlet, radially across the tube.

4. From the top menu of the plot, select "ASCII" and save in desired location. From there you can import data to a program such as Matlab or Excel and plot it in comparison to theoretical values. Close the Comsol Plot, but leave the main screen open.

5. To find the velocity profile at the exit of the tube repeat steps $1-4$, selecting "Velocity Field" as the desired field and using 1e-6 as the z-values. This will produce a plot of the velocity profile at just the one location. By selecting the "keep current plot" option under the "General" tab however, the velocity profiles in other locations can be added.

\section{Part 8: Practice}

1. Calculate $\Delta P$ using the Hagen-Pouseuille law and compare to the value found using the model.

2. Find the Reynolds number to determine whether the flow is laminar or turbulent.

3. Find the velocity profile at the entrance of the tube in order to observe the entrance effect.

4. Rerun the module from the start and do practice problems 1-3 using Vectra A950RX Liquid Crystal Polymer $(\mathrm{n}=0.54, \mathrm{~m}=690)$. 


\section{Example 2: Flow in a Fuel Cell Bipolar Plate}

Note: This example problem will be used in the required course "Transport / Unit Operations 1" which is taken by junior-level chemical engineering students.

Problem Statement: A fuel cell bipolar plate has channels etched in it to allow for the flow of reactant gases. The flow path is rather complicated to allow for equal gas distribution so that there is a uniform fuel concentration at the catalyst. This design will result in better efficiency for the fuel cell. Refer to figure 2.1 below for the simplified geometry that we will be studying here.

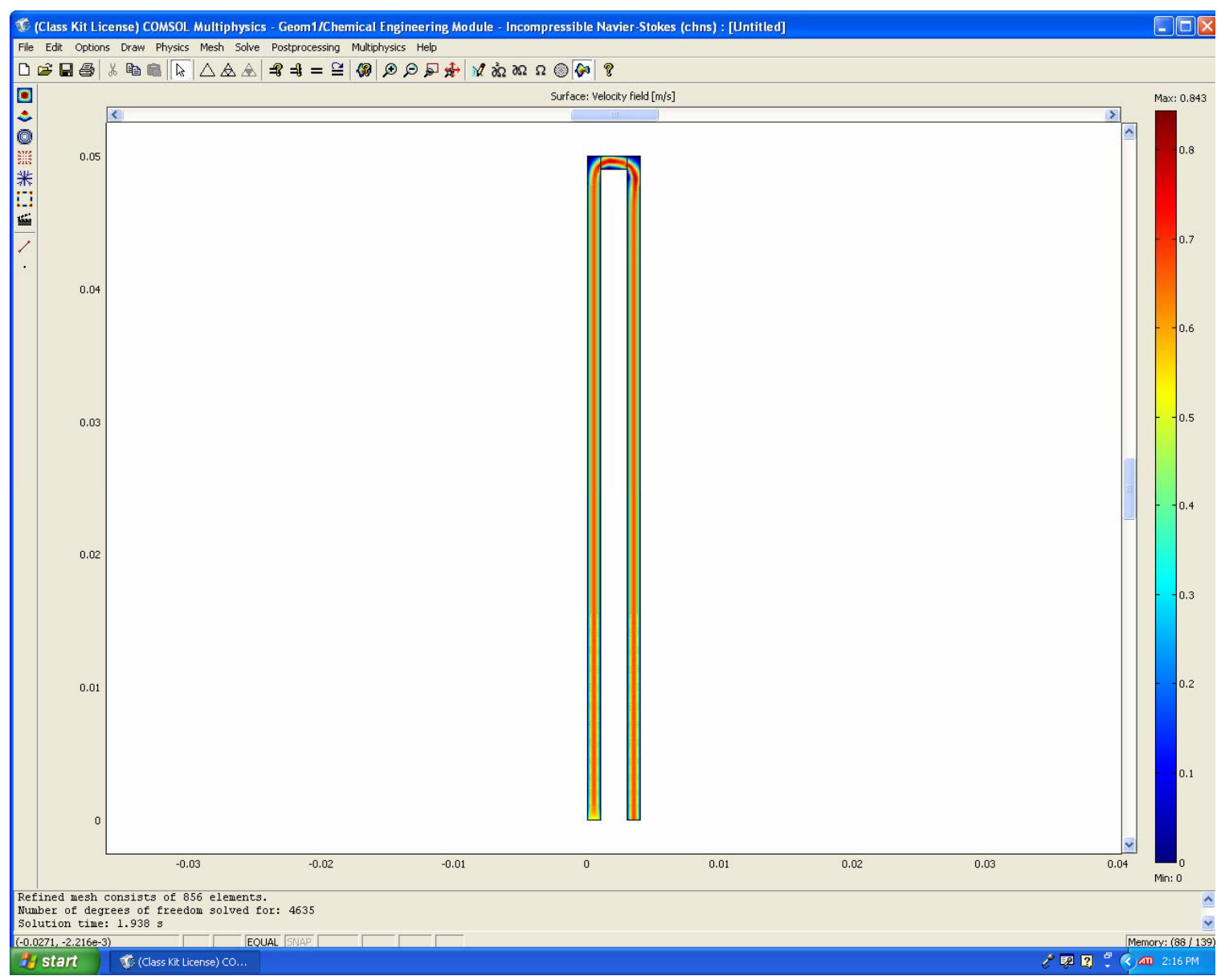

Figure 2.1. Velocity surface plot.

\section{Part 1: Start the program}

1.Start Comsol Multiphysics

2.Within the "New" tab of the "Model Navigator" window there should be a pull-down menu labeled "Space Dimension." Within this pull-down menu select "2D".

3. Expand the folder "Chemical Engineering Module" by clicking the "+" sign to the left of the folder followed by the same for Momentum Balance, Incompressible NavierStokes, and then select Steady-State Analysis. Click "OK." The program should then open to the main screen. 


\section{Part 2: Create the domain}

In this module, a fluid will be modeled flowing through a $U$ shaped tube.

1.Select "Draw" from the tool bar and then "Specify Object" and select rectangle.

2. A box to specify the rectangle should appear on the screen. For this problem we will enter length in meters, time in seconds, and mass in kilograms. Enter 1e-3 for "Width" and $5 \mathrm{e}-2$ for "Height" in the window that appears. Make sure the base is $\mathrm{x}=0$ and $\mathrm{y}=0$. Click "OK."

3. Click the "Zoom Extents" button on the main toolbar. It looks like a red cross with a magnifying glass. This allows the user to view the entire geometry, as seen in the figure on the right above.

4.Once again, select "Draw" from the tool bar and then "Specify Object" and select rectangle. Enter 2e-3 for "Width" and 1e-3 for "Height" in the window that appears. Change the base to $\mathrm{x}=1 \mathrm{e}-3$ and $\mathrm{y}=4.9 \mathrm{e}-2$. Click "OK." You now have a bend in your geometry.

5.Once again, select "Draw" from the tool bar and then "Specify Object" and select rectangle. Enter 1e-3 for "Width" and 5e-2 for "Height" in the window that appears. Change the base to $\mathrm{x}=3 \mathrm{e}-3$ and $\mathrm{y}=0$. Click "OK." You now have a bend in your geometry. Click "OK." You have now finished your bend!

6. While holding down the shift key, click on each rectangle. Then click on the overlapping two red circles on the icons on the left side of the page. This function is called "Union" and joins the rectangles together.

\section{Part 3: Apply physical properties}

1. From the top menu, select Physics, then Subdomain Settings

2. On the left hand side of the pop-up window, there will be a box labeled "Subdomain Selection." In this box select the subdomains 1-3 by holding down the shift key.

3. Enter the dynamic viscosity of 9.6e-6 Pa-s and click "OK." Note that this is a steadystate problem and density is not needed.

\section{Part 4: Apply boundary conditions}

1. From the top menu, select Physics, then Boundary Settings. To specify boundaries highlight a number in the left portion of the box that appears (as shown below).

2. For the bottom of the first rectangle (boundary 2), select a y-velocity of $0.5 \mathrm{~m} / \mathrm{s}$.

3 . For the bottom of the second rectangle (boundary 9), select outflow/pressure $=0 \mathrm{~Pa}$.

4. Make sure all other boundaries are "no slip."

\section{Part 5: Create the mesh}

1. From the top menu, click on the hollow triangle to initialize the mesh. In the bottom left of the screen it should say there are 214 elements in the mesh

2. The button next to "Initialize Mesh" is called "Refine Mesh" (which appears as a triangle within a triangle). This button takes the current mesh and simply makes it more detailed. Click the button once to make the mesh more refined. There should be 856 elements. Please note that problems may occur depending on graphics capabilities of your computer. If this occurs start over but do not refine the mesh. 


\section{Part 6: Solve}

1.Click "Solve" from the pull-down menu and select "Solver Parameters"

2. On the left hand side of the pop-up window there will be a box labeled "Solver:."

Make sure that "Stationary nonlinear" is selected. Click "OK."

3 . From the top menu, click the "=" sign to solve. A window will appear showing how much progress has been made in solving the problem. After the problem is solved and the window disappears, you should see the geometry with a color spectrum indicating a velocity-concentration profile, as seen in the figure below. If you don't see anything, click on "Zoom Extents." This plot is called a Surface Plot. Notice the velocity is zero at the boundary and maximum at the center.

\section{Part 7: Post-Processing}

In this part you will produce a surface plot of the pressure at the inlet and outlet of the channel.

1. From the top menu, select "Postprocessing" then "Plot parameters..."

2. Make sure the "surface" tap is selected, and choose the predefined quantity "Pressure." Select "OK."

3. Verify the maximum pressure of 5.6 Pa.

\section{Part 8: Practice}

1. Calculate a surface plot of velocity and pressure for the geometry shown below which is a first step in a complex bipolar plate geometry. Note there are two separate channels. The "outer" channel is of the same length as in the above module $(50 \mathrm{~mm})$, but the channels are separated by a wider margin. The "inner" channel is $2 \mathrm{~mm}$ shorter than the "outer" channel (thus $48 \mathrm{~mm}$ ). The gap between channels is $1 \mathrm{~mm}$. How would you adjust this system to provide equal velocity in each channel? What would happen if there were a manifold connecting the two inner channels? 


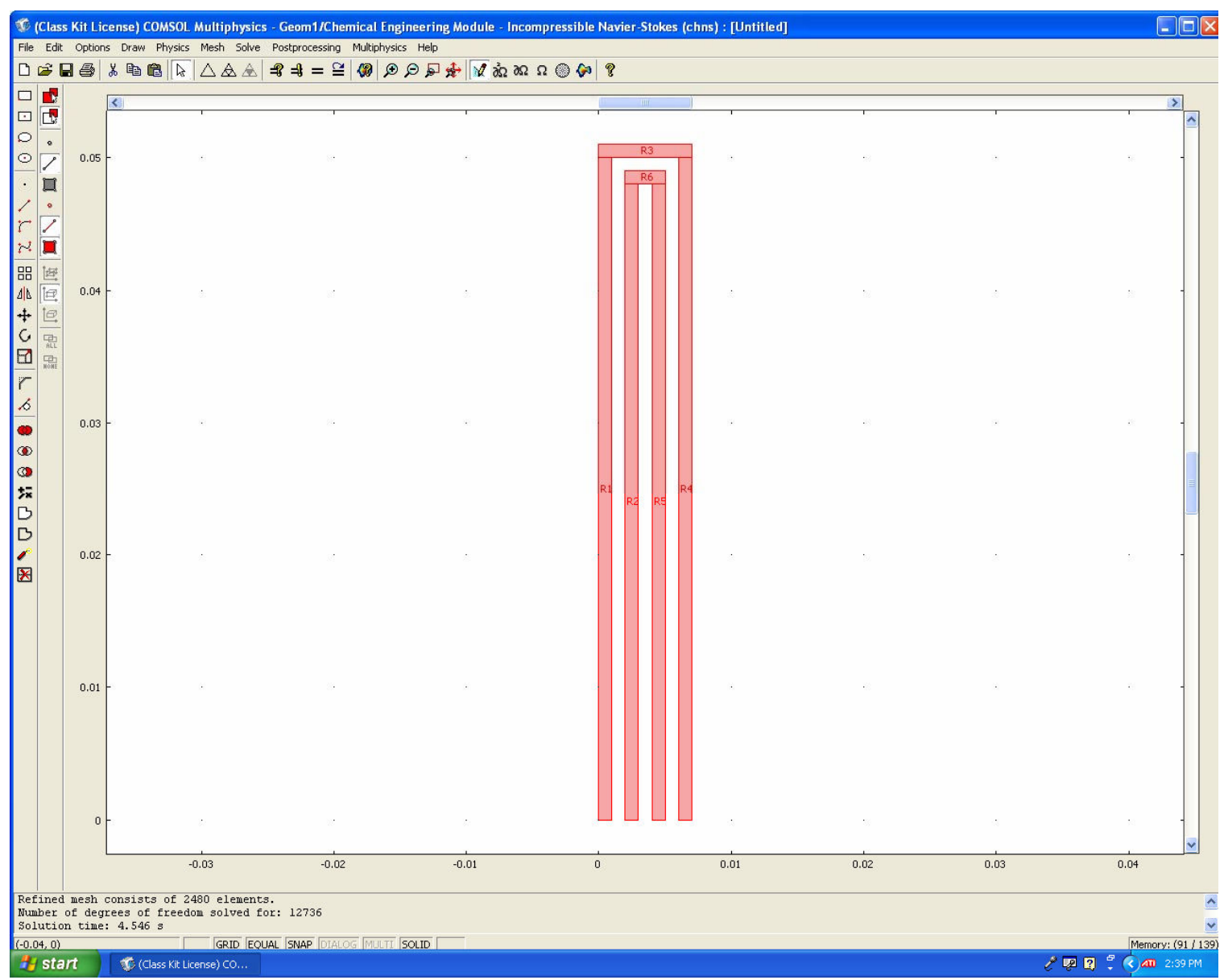

Figure 2.2. Geometry for practice problem.

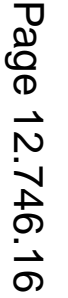




\section{Example 3: Heat Transfer in a Composite Slab with Reaction}

Note: This example problem has been used in an elective course "Fuel Cell Fundamentals" which is taken by chemical, mechanical, electrical, and biomedical engineering students as well as students in materials science. The students range in experience from sophomore to seniors level. Thus this problem is meant to illustrate the ease by which the software can be applied to practical problems.

Problem Statement: Consider heat transfer within a fuel cell bipolar plate. A schematic of the geometry is shown below.

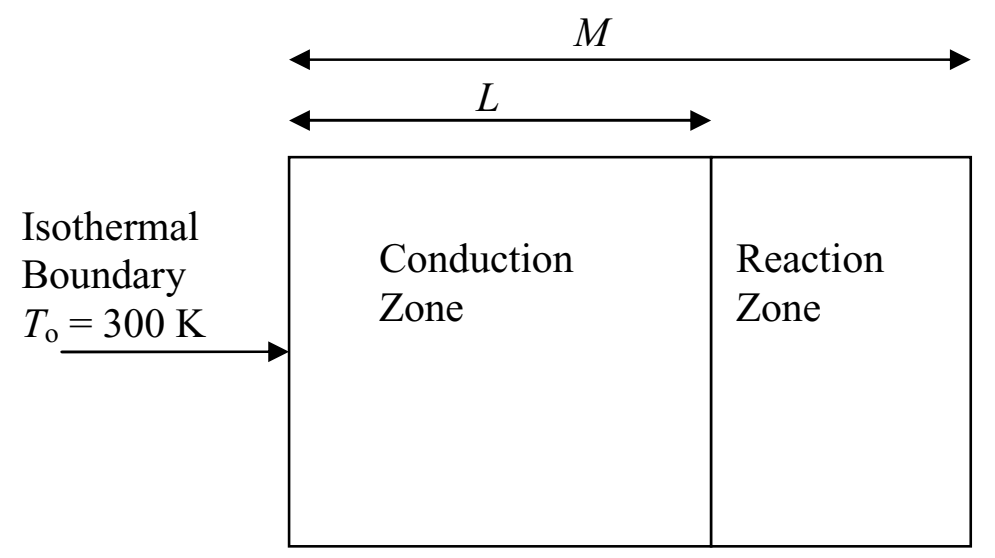

All other external boundaries are insulated and have no heat flux into or out of them. There is continuity of flux and temperature at the conduction zone and reaction zone interface.

The distance from the isothermal boundary to the interface between the conduction and reaction zone is $L$ and the distance from the isothermal boundary to the far edge of the reaction zone is $M$.

The insulated boundaries on the top and bottom essentially make this a one-dimensional heat conduction problem. In the conduction zone, the governing equation is:

$k \frac{\partial^{2} T}{\partial x^{2}}=0$

and in the reaction zone the governing equation is:

$k \frac{\partial^{2} T}{\partial x^{2}}=-Q$ 
where $Q$ is a uniform heat source per unit volume of $100,000,000 \mathrm{~W} / \mathrm{m}^{3}$ within the reaction zone. The value of $k$ is the thermal conductivity and is equal to $20 \mathrm{~W} / \mathrm{m}-\mathrm{K}$ for the bipolar plate. Your task is to find the maximum temperature for the following parameter choices (all distances are in meters)

- $L=0.010, M=0.011$

- $L=0.005, M=0.006$

- $L=0.001, M=0.002$

and compare with the analytical solution given by:

$T_{\max }=T_{o}+\frac{Q}{2 k}\left(M^{2}-L^{2}\right)$

\section{Part 1: Create the domain.}

1. Start the software.

2. Once FEMLAB / Comsol Multiphysics launches, click on the plus sign at the following locations: "Chemical Engineering Module," "Energy Balance," "Conduction," and then select "Steady State Analysis."

3. Click OK

\section{Part 2: Draw the heat transfer geometry}

1. Hold down the shift key and click on the rectangle in the upper left corner of the screen. A window will appear. Insert the following values: For Size use Width $=$ 0.010 , Height $=0.1$. For Position use $\mathrm{x}=0, \mathrm{y}=0$.

2. Click OK

3. Repeat step 1, but insert the following values: For Size use Width $=0.001$, Height $=0.1$. For Position use $\mathrm{x}=0.010, \mathrm{y}=0$.

4. Click OK

5. Click the "Zoom Extends" button on the main toolbar. It looks like a red cross with a magnifying glass.

\section{Part 3: Apply physical properties}

1. From the top menu, select "Physics," then "Subdomain Settings"

2. Select subdomain 1, and enter the thermal conductivity (make sure the isotropic radio button is selected) as 20 (you do not have to enter the heat capacity or thermal conductivity as this is a steady-state problem).

3. Select subdomain 2, and enter the same value for thermal conductivity as in subdomain 1. For heat source, enter a value of $1 \mathrm{e} 8$.

4. Click OK

\section{Part 4: Apply boundary conditions}

1. From the top menu, select "Physics," then "Boundary Settings"

2. Click on boundary number 1 , select "Temperature" from the pull-down menu, and enter 300 .

3. Hold down the shift key, and select boundaries 2, 3, 5, 6, and 7. Make sure it says "Thermal Insulation" on the pull-down menu.

4. Click OK 


\section{Part 5: Create the mesh}

1. Click on the hollow triangle to initialize the mesh.

2. Click on the filled triangle to refine the mesh, once.

\section{Part 6: Solve}

1. Click on "Solve" from the pull-down menu, and select Solver Parameters."

2. Select "Stationary Linear"

3. Click OK

4. Click on "Solve" from the pull-down menu, and select "Solve Problem." You should see the geometry with a color spectrum indicating a temperature profile. If you don't see anything, click on "Zoom Extends."

5. Click on the "Zoom Window" button (looks like a magnifying glass with a red square) to zoom in on the right boundary. Click within the geometry to determine the temperature at the right edge.

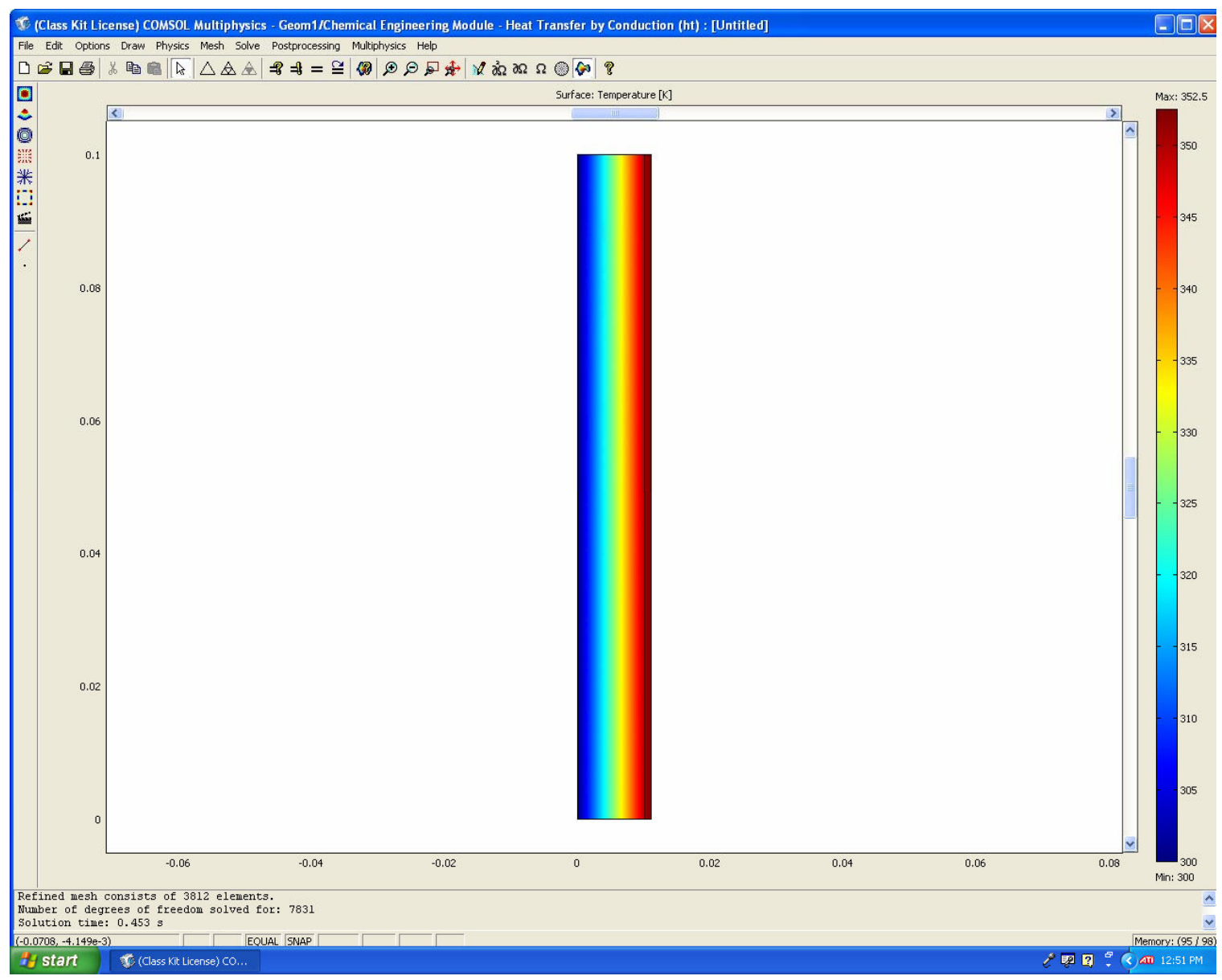

Figure 3.1. Temperature surface plot.

\section{Part 7: Generate plots}

1. Select "Postprocessing" from the pull-down menu, then "Cross-Section Plot Parameters," then select "Line/Extrusion." 
2. For the cross-section line data, enter $\mathrm{x} 0=0.010-L, \mathrm{x} 1=M, \mathrm{y} 0=0.05, \mathrm{y} 1=0.05$. (Note: for the first subproblem with $L=0.010$ and $M=0.011$ you would enter $\mathrm{x} 0$ $=0$ and $\mathrm{x} 1=0.011$ ).

3. Verify a straight line between 0 and $L$ and a parabolic, curved line between $L$ and M.

4. Compare your numbers with the analytical solution.

Repeat for the other 2 geometry selections. This can be done by selecting from the pulldown menu, "Draw," then "Geom 1 (2D)". Double click on the large rectangle and reenter the coordinates as width $=L$ and $\mathrm{x}=0.010-L$. Remesh your geometry and re-run the simulation as outlined above. Note you must enter numbers for width and $\mathrm{x}$ and not the formula given above.

\section{Part 8: Check answers}

- $L=0.010, M=0.011 \quad T_{\max }=352.5^{\circ} \mathrm{C}$

- $L=0.005, M=0.006 \quad T_{\max }=327.5^{\circ} \mathrm{C}$

- $L=0.001, M=0.002 \quad T_{\max }=307.5^{\circ} \mathrm{C}$ 


\section{Example 4: Mass Transfer and Hydrogen Permeation}

Note: This problem has been used in a required junior level chemical engineering course "Transport / Unit Operations 2." Two unsteady-state mass transfer problems are illustrated below.

Problem Statement: Consider mass transfer within a slab. A schematic of the geometry is shown below.

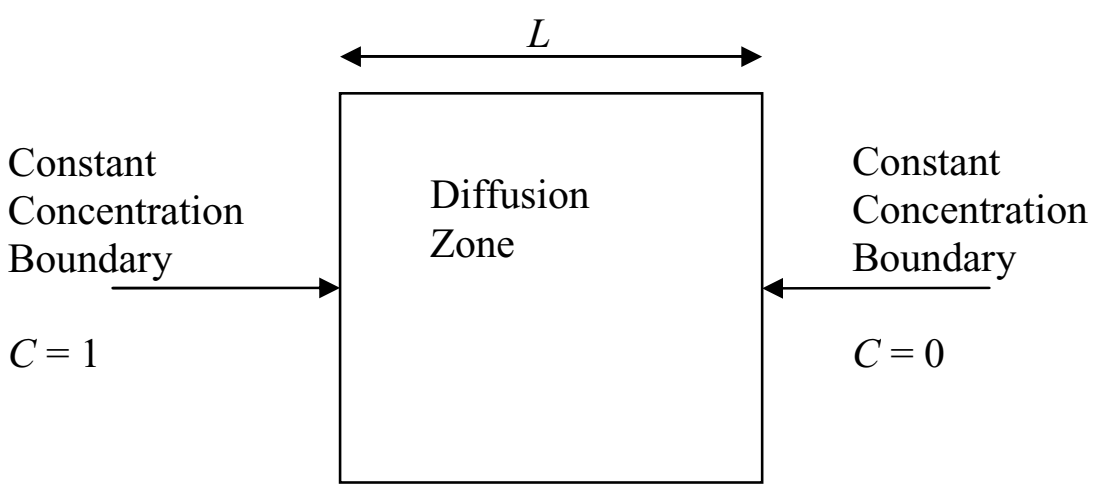

All other external boundaries are insulated and have no mass flux into or out of them. Initially, $C=0$ everywhere.

The distance between boundaries is $L=0.10 \mathrm{~m}$.

The insulated boundaries on the top and bottom essentially make this a one-dimensional mass diffusion problem. In the diffusion zone, the governing equation is:

$$
\frac{\partial C}{\partial t}=D \frac{\partial^{2} C}{\partial x^{2}}
$$

The value of $D$ is the mass diffusivity and is equal to $2.0 \times 10^{-5} \mathrm{~m}^{2} / \mathrm{s}$. Your task is to develop a time-dependent FEMLAB / Comsol Multiphysics model and compare the results of the simulation with the analytical solution. Such work is often done to verify computer code.

With $C_{o}=1$ the analytical solution given by:

$$
\frac{C}{C_{0}}=\left(1-\frac{x}{L}\right)+\sum_{m=1}^{\infty} \frac{-2}{m \pi} \exp \left(\frac{-m^{2} \pi^{2} D t}{L^{2}}\right) \sin \left(\frac{m \pi x}{L}\right)
$$




\section{Part 1: Create the domain}

1. Start the software

2. Once FEMLAB / Comsol Multiphysics launches, click on the plus sign at the following locations: "Chemical Engineering Module," "Mass Balance," "Diffusion," and then select "Transient Analysis."

3. Click OK

\section{Part 2: Draw the mass transfer geometry}

1. Hold down the shift key and click on the rectangle in the upper left corner of the screen. A window will appear. Insert the following values: For Size use Width = 0.10 , Height $=1$. For Position use $\mathrm{x}=0, \mathrm{y}=0$.

2. Click OK

3. Click the "Zoom Extends" button on the main toolbar. It looks like a red cross with a magnifying glass.

\section{Part 3: Apply physical properties}

1. From the top menu, select "Physics," then "Subdomain Settings"

2. Select subdomain 1, and enter the diffusivity (make sure the isotropic radio button is selected) as $2 \mathrm{e}-5$.

3. Select the "init" tab and verify the initial condition is 0 .

4. Click OK

\section{Part 4: Apply boundary conditions}

1. From the top menu, select "Physics," then "Boundary Settings"

2. Click on boundary number 1 , select "Concentration" from the pull-down menu, and enter 1 .

3. Click on boundary number 2, and verify the boundary condition is "Insulation/Symmetry."

4. Click on boundary number 3, and verify the boundary condition is "Insulation/Symmetry."

5. Click on boundary number 4, select "Concentration" from the pull-down menu, and enter 0 .

6. Click OK

\section{Part 5: Create the mesh}

1. Click on the hollow triangle to initialize the mesh.

2. Click on the filled triangle to refine the mesh. There should be 1120 elements.

\section{Part 6: Solve}

1. Click on "Solve" from the pull-down menu, and select Solver Parameters."

2. Select "Time Dependent"

3. Under "Time Stepping" change it to read 0:10:100 (Note these times are in seconds since $D_{A B}$ is in $\mathrm{m}^{2} / \mathrm{s}$.)

4. Click OK

5. Click on "Solve" from the pull-down menu, and select "Solve Problem." You should see the geometry with a color spectrum indicating a concentration profile. 
If you don't see anything, click on "Zoom Extends." This plot is called a "surface plot."

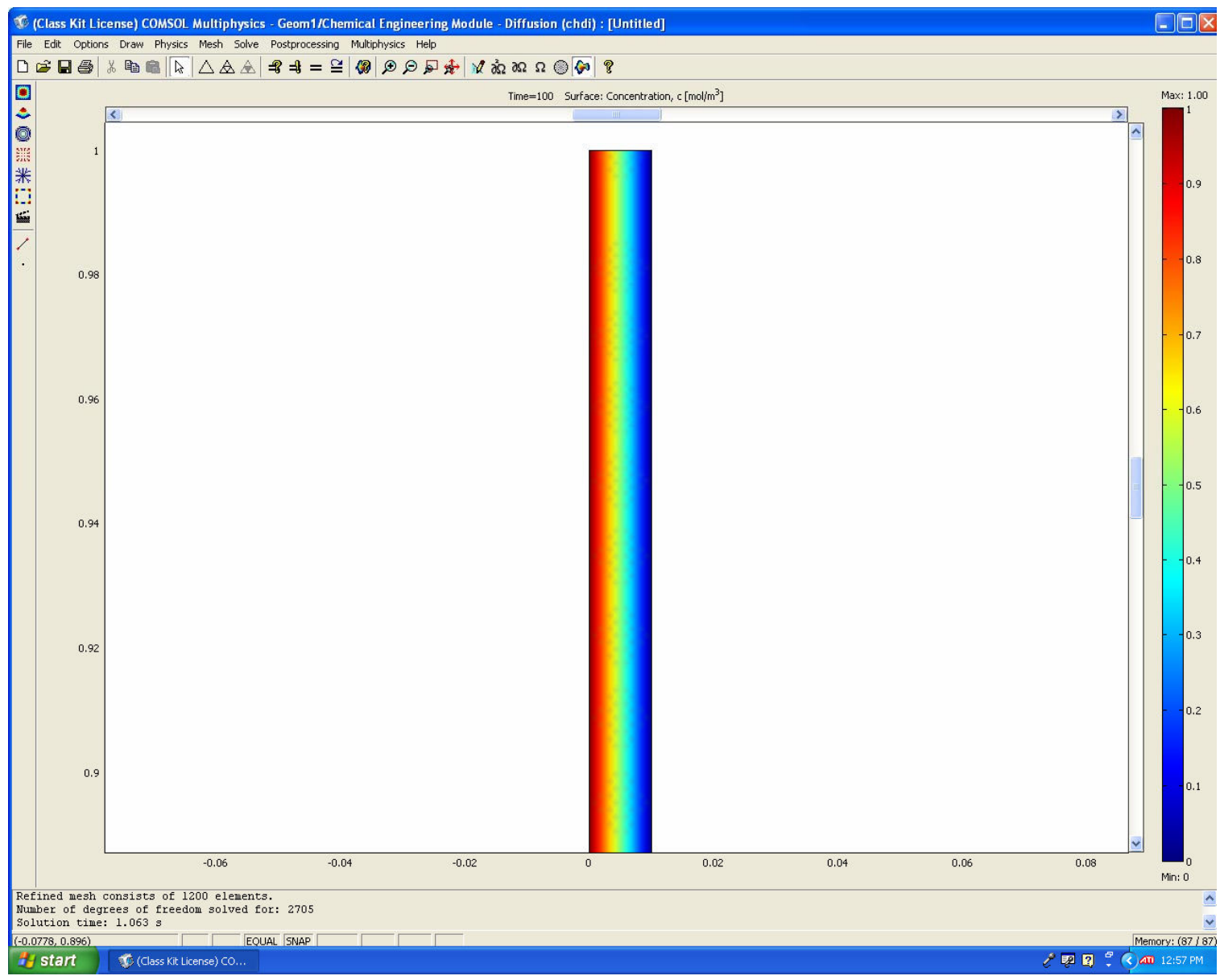

Figure 4.1. Concentration surface plot.

\section{Part 7: Make a color plot}

1. Hit the print screen button

2. Start the program called "Microsoft Paint."

3. Click on "edit," then "paste." You should see the picture of your screen.

4. Go to page setup, select landscape, and fit to 1x1 pages, and print on the color printer.

\section{Part 8: Generate concentration profile}

1. Select "Postprocessing" from the pull-down menu, and pick "Cross-Section Plot Parameters."

2. Select, under the "General" tab, for "Solutions to Use," the solution at $\mathrm{t}=100$ only.

3. Then "Cross-Section Plot Parameters," then select "Line/Extrusion."

4. For the cross-section line data, enter $\mathrm{x} 0=0.0, \mathrm{x} 1=0.1, \mathrm{y} 0=0.5, \mathrm{y} 1=0.5$.

5. On the plot, click on the letters "ASCII" and enter a file name to save the data. Make sure you put it in a folder that you can find later. 
6. Within MATLAB or EXCEL, plot this data at increments of $\Delta \mathrm{x}=0.01 \mathrm{~m}$ (thus at $\mathrm{x}=0.00,0.01,0.02$, etc. up to 0.10 ). Plot the data as some sort of symbol (either an $\mathrm{x}$, star, square, etc.)

7. Calculate the Fourier number for this problem, $\mathrm{Dt} / \mathrm{L}^{2}$. If this number is greater than 0.1 you can probably use just one term in the analytical series solution.

8. In the same graph that you created in step 6, plot the analytical solution as a line. Note to have a smooth plot, you should use a smaller $\Delta \mathrm{x}$.

\section{Part 9: Practice}

Consider the cross-section of the bipolar plate, shown below:

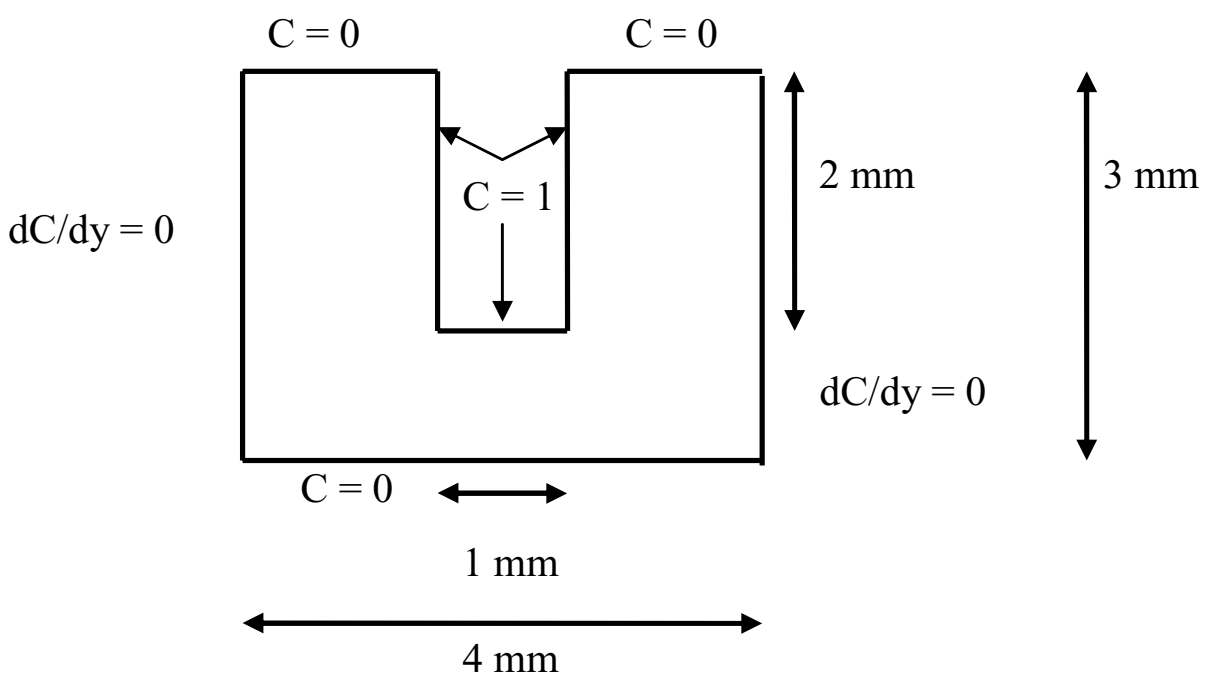

At the top and bottom of the plate, the concentration is always zero. At the left and right of the plate, there is "symmetry" from the region surrounding one channel to the next, so the slope in the concentration is zero. Within the channel itself, the concentration at the plate / channel interface is one. The initial concentration is zero. Note you can use the "difference button" to help you make this geometry.

Note that the diffusion coefficient is $\mathrm{D}_{\mathrm{AB}}=10^{-5} \mathrm{~mm}^{2} / \mathrm{s}$. (What is this in $\mathrm{m}^{2} / \mathrm{s}$ ?)

Use FEMLAB / Comsol Multiphysics to determine a "surface plot" of the concentration after $10000 \mathrm{~s}$. 


\section{Example 5: Transport Effects on Kinetics}

Note: This problem can be used in a required junior level chemical engineering course "Transport / Unit Operations 2." Three steady-state mass transfer problems with reaction are illustrated below.

Problem Statement: Consider the problem of species diffusion and first order reaction within a catalyst "cube" of length $L$. A schematic of the geometry is shown below.

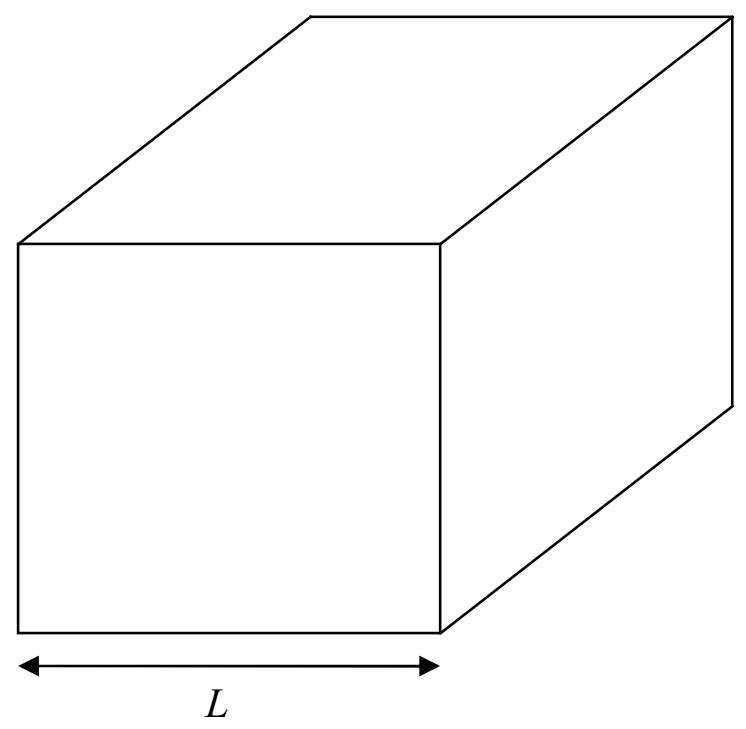

All external boundaries have constant concentration $C=C_{o}$.

Within the cube, the governing equation is:

$D\left(\frac{\partial^{2} C}{\partial x^{2}}+\frac{\partial^{2} C}{\partial y^{2}}+\frac{\partial^{2} C}{\partial z^{2}}\right)=k C$

where $D$ is the species diffusivity and $k$ is the reaction rate coefficient. In dimensionless terms, choosing $C^{*}=C / C_{0}, x^{*}=x / L, y^{*}=y / L$, and $z^{*}=z / L$, and defining the Thiele modulus as $\phi^{2}=k L^{2} / D$ we have:

$\left(\frac{\partial^{2} C}{\partial x^{2}}+\frac{\partial^{2} C}{\partial y^{2}}+\frac{\partial^{2} C}{\partial z^{2}}\right)=\phi^{2} C$

Your task is to find the effectiveness factor for the following parameter choices for $\phi^{2}$ : $0.1,1.0$, and 10.0. 


\section{Part 1: Create the domain}

1. Start FEMLAB / Comsol Multiphysics.

2. Once FEMLAB launches, select 3D from the "Space Dimension" pull-down menu.

3. Click on the plus sign at the following locations: "Chemical Engineering Module," "Mass Balance," "Diffusion," and then select "Steady State Analysis."

4. Click OK

\section{Part 2: Draw the mass transfer geometry}

1. Hold down the shift key and click on the block in the upper left corner of the screen. A window will appear. Insert the following values: Style: Solid, Base: Corner, Axis Base Point: $\mathrm{x}=0, \mathrm{y}=0, \mathrm{z}=0$, Length: $\mathrm{x}=1, \mathrm{y}=1$, and $\mathrm{z}=1$.

2. Click OK

3. Click the "Zoom Extends" button on the main toolbar. It looks like a red cross with a magnifying glass.

\section{Part 3: Apply physical properties}

1. From the top menu, select "Physics," then "Subdomain Settings"

2. Select subdomain 1, and enter the diffusivity (make sure the isotropic radio button is selected) as 1.0 and the reaction rate as $-0.1 * \mathrm{c}$.

3. Click OK

\section{Part 4: Apply boundary conditions}

1. From the top menu, select "Physics," then "Boundary Settings"

2. Select all boundaries 1-6, select "Concentration" from the pull-down menu, and enter 1.

3. Click OK

\section{Part 5: Create the mesh}

1. Click on the hollow triangle to initialize the mesh. There should be about 18,000 elements!

\section{Part 6: Solve}

1. Click on "Solve" from the pull-down menu, and select Solver Parameters."

2. Select "Stationary Linear"

3. Click OK

4. Click on "Solve" from the pull-down menu, and select "Solve Problem." You should see the geometry with a color spectrum indicating a temperature profile. If you don't see anything, click on "Zoom Extends." Have some fun rotating the geometry around. Make sure it is a "slice plot."

5. Write down the minimum concentration. 


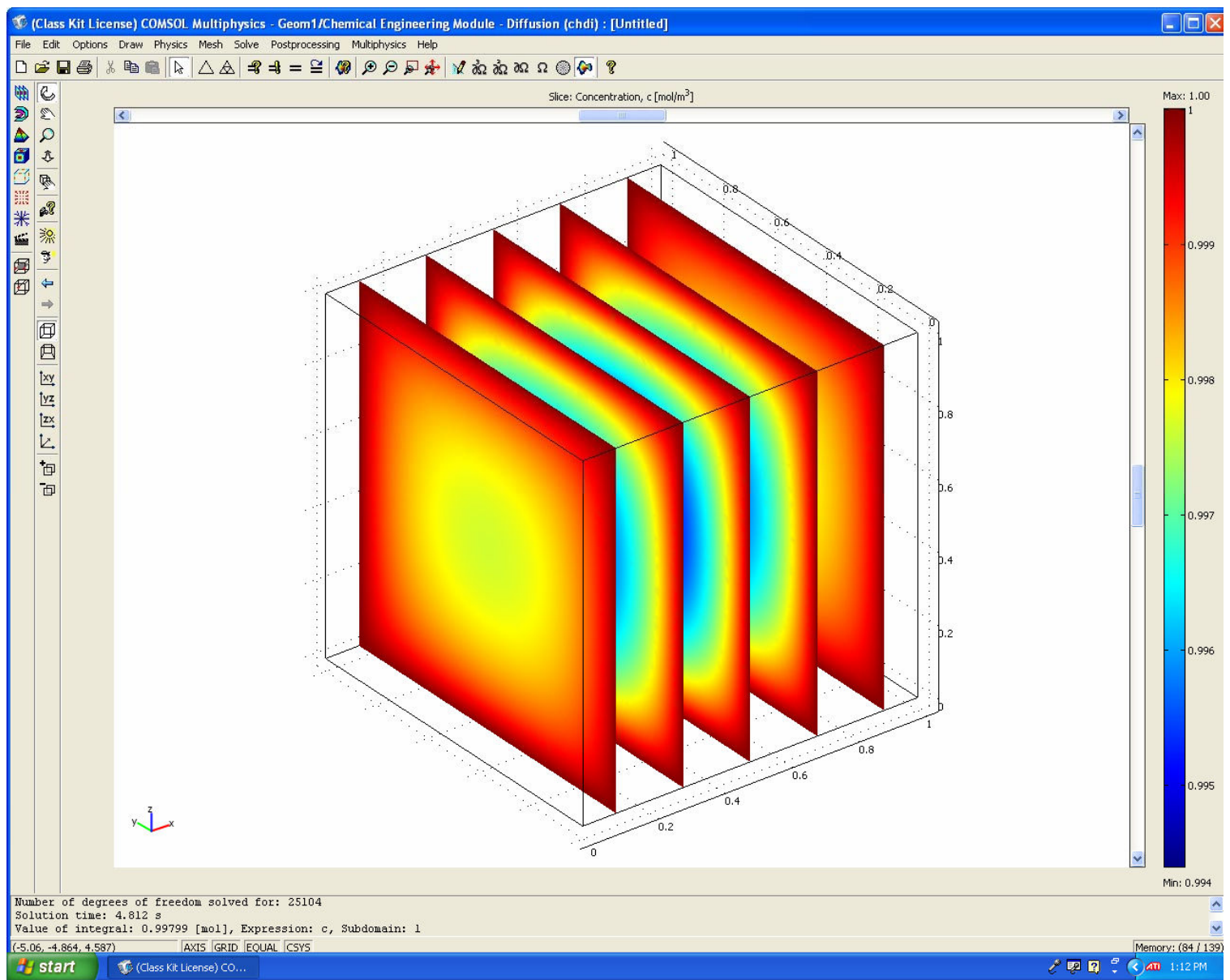

Figure 5.1. Concentration surface plot.

\section{Part 7: Generate plots}

The effectiveness factor is defined according to the integral $\eta=\frac{k \iiint C d x d y d z}{k C_{o} L^{3}}$

In terms of our dimensionless variables, this is given as:

$\eta=\iiint C^{*} d x^{*} d y^{*} d z^{*}$

1. This is easily accomplished within FEMLAB. Select "Postprocessing" from the pull-down menu, then "Subdomain Integration" then select OK.

2. Write down your result for the effectiveness factor.

Repeat for the other 2 parameter selections, when $\phi^{2}=1.0$ and 10.0.

Note that in class we used the half width of the catalyst "slab" to render the system dimensionless. Here, we use the entire length of the cube. Thus, the results cannot really be compared with each other without making some adjustments. 


\section{Part 8: Check answers}

- $\phi^{2}=0.1, C_{\min }=0.994, \eta=0.997$.

- $\phi^{2}=1.0, C_{\min }=0.946, \eta=0.980$.

- $\phi^{2}=10.0, C_{\text {min }}=0.601, \eta=0.845$.

\section{Part 9: Practice}

For what value of $\phi^{2}$ does $\eta=0.5$ ? (Hint: $\phi^{2}$ is a multiple of 10.) What is the minimum concentration under these conditions? Also try to get $\eta$ for a "cone catalyst pellet", with radius $=1$, height $=1$, and semiangle $=45$, with $\phi^{2}=10$. 